\title{
Quasi-periodic solutions for second order differential equation with superlinear asymmetric nonlinearities and nonlinear damping term
}

Xiaoming Wang ${ }^{*}$

*Correspondence:

wxmsuda03@163.com Chern Institute of Mathematics, Nankai University, Tianjin, 300071 People's Republic of China Department of Mathematics, Shangrao Normal University, Shangrao, Jiangxi 334001, People's Republic of China

\begin{abstract}
In this paper, the existence of Aubry-Mather sets and quasi-periodic solutions of the oscillator $x^{\prime \prime}+\alpha x^{+3}-\beta x^{-3}+f(x) q\left(x^{\prime}\right)+\psi(x)=p(t)$ are established, where $f, q$, and $\psi$ belong to the class $C^{1}(R), p$ is a continuous $2 \pi$-periodic function. Under some assumptions on the parities of $f, \psi$, and $p$, we prove that there are infinitely many generalized quasi-periodic solutions by a result of Shuinee Chow and Mingliang Pei from the Aubry-Mather theorem of reversible mappings.
\end{abstract}

MSC: $34 C 15 ; 37 C 55$

Keywords: reversible systems; quasi-periodic solutions; asymmetric oscillator; Aubry-Mather sets

\section{Introduction and main result}

In this paper, we are concerned with the existence of Aubry-Mather sets and quasiperiodic solutions to the following second order differential equation with superlinear asymmetric nonlinearities and nonlinear damping term:

$$
x^{\prime \prime}+\alpha x^{+3}-\beta x^{-3}+f(x) q\left(x^{\prime}\right)+\psi(x)=p(t)
$$

where $x^{ \pm}=\max \{ \pm x, 0\}, \alpha$ and $\beta$ are strictly positive real numbers. We assume the functions $f, q$, and $\psi$ belong to the class $C^{1}(\mathbb{R})$ and $p(t) \in C^{0}\left(\mathbf{S}^{1}\right)$ is a $2 \pi$-periodic continuous function, where $\mathbf{S}^{1}=\mathbb{R} / 2 \pi \mathbb{Z}$.

In the last two decades, there has been an increasing interest in obtaining sufficient conditions for the existence of Aubry-Mather sets and quasi-periodic solutions due to such solutions providing a rather complete qualitative description of the dynamics, for different classes of nonlinear second order differential equations independent of a damping term, or with a damping term. We refer to [1-17], and references therein. For example, in [10], Liu and Wang have studied the following nonlinear Liénard equations:

$$
x^{\prime \prime}+f(x) x^{\prime}+n^{2} x+\psi(x)=p(t)
$$

\section{Springer}


where $f(x)$ and $\psi(x)$ are $C^{2}$ smooth in $x, p \in C^{2}\left(\mathbf{S}^{1}\right)$ is a $2 \pi$-periodic function and $n$ is a positive integer. They obtained the existence of Aubry-Mather sets and quasi-periodic solutions based on the well-known Aubry-Mather theorem for reversible systems due to Chow and Pei [8], who gave some sufficient conditions for the existence of Aubry-Mather sets for some planar reversible maps with a linear damping term as well.

However, to the best of our knowledge, the existence of Aubry-Mather sets and quasiperiodic solutions, when the damping term comprises a nonlinear function of $x^{\prime}$, have been relatively little researched. In [7], Capietto et al. considered the equation with semilinear asymmetric term and a nonlinear damping term of the form

$$
x^{\prime \prime}+\alpha x^{+}-\beta x^{-}+\psi(x)+h\left(t, x^{\prime}, x\right)=p(t)
$$

where $\psi, p$ belong to the class $C^{2}(\mathbb{R})$ and $h \in C^{2}\left(\mathbb{R}^{3}\right)$. Moreover, $p$ and $h$ are $2 \pi$-periodic in the time variable. Under some symmetry assumptions on $\psi, p$ and $h$, the differential equation (1.3) has a reversible structure. By an Aubry-Mather theorem for reversible mapping, due to Chow and Pei [8], one obtained the existence of Aubry-Mather sets and quasiperiodic solutions of (1.3) in the resonant case

$$
\frac{1}{\sqrt{\alpha}}+\frac{1}{\sqrt{\beta}}=\frac{2}{n}
$$

where $n$ is a positive integer.

In this paper, replacing the semilinear asymmetric term $\alpha x^{+}-\beta x^{-}$in (1.3) by a superlinear asymmetric term $\alpha x^{+3}-\beta x^{-3}$, and as $h\left(t, x^{\prime}, x\right)=f(x) q\left(x^{\prime}\right)+\psi(x)$ in (1.3), we will investigate the existence of Aubry-Mather sets and quasi-periodic solutions for (1.1). This equation models the motion of a particle subject to an asymmetric restoring force (see e.g. [11]) and a damping force. In the present paper, we also assume that the system (1.1) is a reversible system. By proposing a new estimate approach and borrowing a new analytical trick from the recent papers $[16,18,19]$ by the present author, we will show that under some reasonable assumptions, (1.1) has quasi-periodic solutions in generalized sense, that is, the Poincaré map of (1.1) has Aubry-Mather sets. Especially, the smoothness assumption on functions $f, q, \psi$ only belong to $C^{1}(\mathbb{R})$ and $p(t)$ only belongs to $C^{0}\left(\mathbf{S}^{1}\right)$. The results of this paper are new and they complement previously known results.

In what follows, we tacitly assume that

$\left(\mathrm{H}_{1}\right) f(x), q(x), \psi(x) \in C^{1}(\mathbb{R}), p(t) \in C^{0}\left(\mathbf{S}^{1}\right)$;

$\left(\mathrm{H}_{2}\right) f(-x)=-f(x), \psi(-x)=-\psi(x), p(-t)=-p(t)$;

$\left(\mathrm{H}_{3}\right)$ there exists a constant $\mu>0$, such that

$$
\left|x \psi^{\prime}(x)\right| \leq \mu, \quad \forall x \in \mathbb{R}
$$

$\left(\mathrm{H}_{4}\right) \lim _{|x| \rightarrow+\infty} \sup \left|\frac{x f^{\prime}(x)}{x^{\gamma}}\right|<+\infty$, where $\gamma \in(0,1)$;

$\left(\mathrm{H}_{5}\right)$ there exists a constant $\sigma \in\left(0, \frac{1}{2}\right)$, such that

$$
\lim _{|x| \rightarrow+\infty} \sup \left|\frac{x q^{\prime}(x)}{x^{\sigma}}\right|<+\infty, \quad \text { where } \sigma \text { further satisfies } 2 \sigma+\gamma<1
$$


Remark 1.1 By $\left(\mathrm{H}_{3}\right)$ and the rule of L'Hospital, we have

$$
\lim _{|x| \rightarrow+\infty} \psi^{\prime}(x)=0 \quad \text { and } \quad \lim _{|x| \rightarrow+\infty} \frac{\psi(x)}{x}=0
$$

Remark 1.2 Hypothesis $\left(\mathrm{H}_{4}\right)$ implies there exists $a>0$ such that

$$
\left|x f^{\prime}(x)\right| \leq a\left(1+|x|^{\gamma}\right), \quad|f(x)| \leq a\left(1+|x|^{\gamma}\right), \quad \text { for all } x \in \mathbb{R}
$$

Remark 1.3 From $\left(\mathrm{H}_{5}\right)$, it is easy to see that there exists $b>0$ such that

$$
\left|x q^{\prime}(x)\right| \leq b\left(1+|x|^{\sigma}\right), \quad|q(x)| \leq b\left(1+|x|^{\sigma}\right), \quad \text { for all } x \in \mathbb{R} .
$$

The main result of this work is the following.

Theorem 1.1 Suppose $\left(\mathrm{H}_{1}\right)-\left(\mathrm{H}_{5}\right)$ and $\psi^{\prime}(0)=f^{\prime}(0)=q^{\prime}(0)=0$ hold. Then there exists $\lambda_{0}>0$, such that for any $\lambda \in\left(\lambda_{0},+\infty\right)$, (1.1) possesses a solution $z_{\lambda}(t)=\left(x_{\lambda}(t), x_{\lambda}^{\prime}(t)\right)$ of Mather type with rotation number $\lambda$, that is,

(i) if $\lambda=\frac{n}{m}$ is rational, and $(n, m)=1$, the solutions $z_{\lambda}^{i}(t)=z_{\lambda}(t+2 \pi i), 0 \leq i \leq m-1$, are mutually unlinked periodic solutions of period $2 \pi m$;

(ii) if $\lambda$ is irrational, the solution $z_{\lambda}(t)$ is either a usual quasi-periodic solution or a generalized one.

Remark 1.4 A solution is called generalized quasi-periodic if the closed set

$$
M_{\lambda} \equiv\left\{\overline{z_{\lambda}(2 \pi i), i \in \mathbb{Z}}\right\}
$$

is a Denjoy minimal set.

Remark 1.5 In this work, we remark that our estimate methods of the Poincaré map associated to the planar system equivalent to (1.1) are different from those used in [7] and [10] to some degree.

Remark 1.6 Theorem 1.1 in [16] can be regarded as a direct generalization in the present paper when $f(x)=0$.

Example 1.1 The conclusions of Theorem 1.1 hold if we let $f(x)=\frac{x^{\frac{5}{3}}}{1+x^{\frac{4}{3}}} \in C^{1}(\mathbb{R}), q(x)=$ $x^{\frac{1}{5}} \arctan x \in C^{1}(\mathbb{R}), \psi(x)=\arctan x^{3} \in C^{1}(\mathbb{R})$, and $\forall p(t) \in C^{0}\left(\mathbf{S}^{1}\right)$, and we take the constants $\mu=2, \sigma=\frac{1}{5}, \gamma=\frac{1}{3}, a=\frac{5}{3}, b=2$ in $\left(\mathrm{H}_{1}\right)-\left(\mathrm{H}_{5}\right)$.

The rest of paper is organized as follows. In Section 2, we introduce the action-angle variables to transform the system (1.1) into a perturbation of an integrable system, and then give some growth estimates on the corresponding action and angle variables functions. In Section 3, we give some crucial estimates by some lemmas which say that the Poincaré mapping of the new system is close to the so-called twist map around infinity. Then the Aubry-Mather theorem for reversible systems developed by Chow and Pei [8] guarantees the existence of Aubry-Mather sets and quasi-periodic solutions for (1.1). 


\section{Action-angle variables and some properties}

In this section, we will transform (1.1) into another system expressed in action-angle variables.

Let $x^{\prime}=y$. Then (1.1) is equivalent to the following system:

$$
x^{\prime}=y, \quad y^{\prime}=-f(x) q(y)-\alpha x^{+3}+\beta x^{-3}-\psi(x)+p(t) .
$$

From assumption $\left(\mathrm{H}_{2}\right)$, we know that (2.1) is reversible under the involution $(x, y) \mapsto$ $(-x, y)$. For the definition of reversible system and further properties of reversible system, see Appendix A of [13].

In order to make an action-angle transformation, we consider the auxiliary system

$$
x^{\prime}=y, \quad y^{\prime}=-\alpha x^{+3}+\beta x^{-3}
$$

with the Hamiltonian

$$
H(x, y)=\frac{1}{2} y^{2}+\frac{\alpha}{4} x^{+4}+\frac{\beta}{4} x^{-4} .
$$

Clearly, $H>0$ on $\mathbb{R}^{2}$ except at the only equilibrium point $(x, y)=(0,0)$ where $H=0$.

Let $(C(t), S(t))$ be the solution of (2.2) satisfying the initial condition $(C(0), S(0))=(1,0)$ and let $T>0$ be its minimal period. From (2.2), we can find that $C(t)$ and $S(t)$ satisfy the following properties.

\section{Lemma 2.1}

(i) $C^{\prime}(t)=S(t), S^{\prime}(t)=-\alpha C^{+}(t)^{3}+\beta C^{-}(t)^{3}$;

(ii) $2 S(t)^{2}+\alpha C^{+}(t)^{4}+\beta C^{-}(t)^{4} \equiv \alpha$;

(iii) $C(-t)=C(t), S(-t)=-S(t)$;

(iv) $|C(t)| \leq \max \left\{1, \sqrt[4]{\frac{\alpha}{\beta}}\right\}:=C_{\infty},|S(t)| \leq \sqrt{\frac{\alpha}{2}}:=S_{\infty}$.

The action and angle variables are now defined by the mapping $\Psi: \mathbb{R}^{+} \times \mathbf{S}^{1} \rightarrow \mathbb{R}^{2} \backslash\{0\}$, $(x, y)=\Psi(I, \theta)$ being defined by the formula

$$
x=\lambda^{\frac{1}{3}} I^{\frac{1}{3}} C\left(\frac{\theta}{\omega}\right), \quad y=\lambda^{\frac{2}{3}} I^{\frac{2}{3}} S\left(\frac{\theta}{\omega}\right),
$$

where $\omega=\frac{2 \pi}{T}, \lambda=\frac{3 \omega}{\alpha T}$ are constants. By some simple calculations, it is easy to see that $\frac{\partial x}{\partial I}=\frac{x}{3 I}, \frac{\partial x}{\partial \theta}=\frac{y}{\omega \lambda^{\frac{1}{3}} I^{\frac{1}{3}}}, \frac{\partial y}{\partial I}=\frac{2 y}{3 I}, \frac{\partial y}{\partial \theta}=\frac{-\alpha x^{+3}+\beta x^{-3}}{\omega \lambda^{\frac{1}{3}} I^{\frac{1}{3}}}$. We claim that $\Psi$ is a symplectic diffeomorphism from $\mathbb{R}^{+} \times \mathbf{S}^{1}$ onto $\mathbb{R}^{2} \backslash\{0\}$. Indeed, the Jacobian determinant of $\Psi$ is 1, so $\Psi$ is measure preserving. Moreover, since $(C(t), S(t))$ is a solution of (2.2) and has $T$ as its minimal period, one concludes that $\Psi$ is one to one and onto. This finishes the claim.

Under $\Psi$, the system (2.1) is transformed into

$$
\dot{\theta}=\Phi_{1}(t, \theta, I), \quad \dot{I}=\Phi_{2}(t, \theta, I),
$$

where

$$
\Phi_{1}(t, \theta, I)=\frac{1}{3} \lambda^{\frac{4}{3}} \alpha I^{\frac{1}{3}}+\frac{x(\theta, I)(f(x(\theta, I)) q(y(\theta, I))+\psi(x(\theta, I))-p(t))}{3 I},
$$




$$
\Phi_{2}(t, \theta, I)=-\frac{y(\theta, I)(f(x(\theta, I)) q(y(\theta, I))+\psi(x(\theta, I))-p(t))}{\omega \lambda^{\frac{1}{3}} I^{\frac{1}{3}}} .
$$

According to our symmetry assumptions and the definition of reversible system, we note that system (2.4) and its Poincaré map are reversible in $\theta$ with respect to the involution $(\theta, I) \mapsto(-\theta, I)$.

We observe that the relation between (2.4) and (2.1) is that if $\theta(t)=\theta\left(t ; \theta_{0}, I_{0}\right), I(t)=$ $I\left(t ; \theta_{0}, I_{0}\right)$ are the solution of (2.4) with the initial value condition $\theta(0)=\theta_{0}, I(0)=I_{0}$, then

$$
x\left(t ; \theta_{0}, I_{0}\right)=x\left(\theta\left(t ; \theta_{0}, I_{0}\right), I\left(t ; \theta_{0}, I_{0}\right)\right)=\lambda^{\frac{1}{3}} I^{\frac{1}{3}}\left(t ; \theta_{0}, I_{0}\right) C\left(\frac{\theta\left(t ; \theta_{0}, I_{0}\right)}{\omega}\right)
$$

and

$$
y\left(t ; \theta_{0}, I_{0}\right)=y\left(\theta\left(t ; \theta_{0}, I_{0}\right), I\left(t ; \theta_{0}, I_{0}\right)\right)=\lambda^{\frac{2}{3}} I^{\frac{2}{3}}\left(t ; \theta_{0}, I_{0}\right) S\left(\frac{\theta\left(t ; \theta_{0}, I_{0}\right)}{\omega}\right)
$$

are the solutions of $(2.1)$ with initial data $x(0)=x\left(0 ; \theta_{0}, I_{0}\right), y(0)=y\left(0 ; \theta_{0}, I_{0}\right)$.

For the sake of convenience, in later discussions we will replace $\theta\left(t ; \theta_{0}, I_{0}\right), I\left(t ; \theta_{0}, I_{0}\right)$, $x\left(\theta\left(t ; \theta_{0}, I_{0}\right), I\left(t ; \theta_{0}, I_{0}\right)\right), y\left(\theta\left(t ; \theta_{0}, I_{0}\right), I\left(t ; \theta_{0}, I_{0}\right)\right)$ by $\theta, I, x, y$, respectively.

Now we will provide some information on the growth behavior of $I\left(t ; \theta_{0}, I_{0}\right)$ and $\theta\left(t ; \theta_{0}, I_{0}\right)$ in the following two lemmas.

Lemma 2.2 The limit

$$
\lim _{I_{0} \rightarrow+\infty} I\left(t ; \theta_{0}, I_{0}\right)=+\infty
$$

holds uniformly on $t \in[0,2 \pi]$.

Proof From $\left(\mathrm{H}_{3}\right)-\left(\mathrm{H}_{5}\right)$ and (2.3), there exist constants $r_{1}>0, r_{2}>0$, such that

$$
|\dot{I}(t)|=\left|-\frac{y(f(x) q(y)+\psi(x)-p(t))}{\omega \lambda^{\frac{1}{3}} I^{\frac{1}{3}}}\right| \leq r_{1} I(t)+r_{2}, \quad I \neq 0 .
$$

Then, by the Gronwall inequality, we have

$$
e^{-2 \pi r_{1}} I_{0}-\frac{r_{2}}{r_{1}}\left(1-e^{-2 \pi r_{1}}\right) \leq I(t) \leq e^{2 \pi r_{1}} I_{0}+\frac{r_{2}}{r_{1}}\left(e^{2 \pi r_{1}}-1\right)
$$

for all $t \in[0,2 \pi]$.

So, by $(2.5), I\left(t ; \theta_{0}, I_{0}\right) \rightarrow+\infty$ as $I_{0} \rightarrow+\infty$ uniformly for $t \in[0,2 \pi]$.

Lemma 2.3 There exist constants $k_{2}>k_{1}>0$ and $\bar{I}>0$, such that for any $I_{0} \geq \bar{I}$, we have

(i) $k_{1} I_{0} \leq I\left(t ; \theta_{0}, I_{0}\right) \leq k_{2} I_{0}$, for $\forall \theta_{0} \in \mathbb{R}$ and $\forall t \in[0,2 \pi]$.

(ii) $\theta^{\prime}\left(t ; \theta_{0}, I_{0}\right)>0$, for $\forall \theta_{0} \in \mathbb{R}$ and $\forall t \in[0,2 \pi]$.

Proof (i) By the inequality (2.5), we can easily find constants $k_{2}>k_{1}>0$ and $\bar{I}>0$, such that

$$
k_{1} I_{0} \leq I(t) \leq k_{2} I_{0}
$$

for any $I_{0} \geq \bar{I}$ and $\forall t \in[0,2 \pi]$. 
(ii) From Remark 1.1, we know $\lim _{|x| \rightarrow+\infty} \frac{\psi(x)}{x}=0$, then for every $\varepsilon>0$, there exists $M=$ $M(\varepsilon) \geq 0$, such that

$$
|\psi(x)| \leq \varepsilon|x|
$$

if $|x| \geq M$ and $\forall t \in[0,2 \pi]$. Hence, from Remark 1.2 and Remark 1.3, we have

$$
\begin{aligned}
\frac{d \theta}{d t}= & \frac{1}{3} \lambda^{\frac{4}{3}} \alpha I^{\frac{1}{3}}+\frac{x(f(x) q(y)+\psi(x)-p(t))}{3 I} \\
\geq & \frac{1}{3} \lambda^{\frac{4}{3}} \alpha I^{\frac{1}{3}}-\frac{|x f(x) q(y)|}{3 I}-\frac{|x \psi(x)|+|p(t) x|}{3 I} \\
\geq & \frac{1}{3} \lambda^{\frac{4}{3}} \alpha I^{\frac{1}{3}}-\frac{a b|x|\left(1+|x|^{\gamma}\right)\left(1+|y|^{\sigma}\right)}{3 I}-\frac{\varepsilon x^{2}+p_{\infty}|x|}{3 I} \\
\geq & \frac{1}{3} \lambda^{\frac{4}{3}} \alpha I^{\frac{1}{3}}-\frac{\left(p_{\infty}+a b\right) \lambda^{\frac{1}{3}} C_{\infty}}{3 I^{\frac{2}{3}}}-\frac{a b \lambda^{\frac{3+\gamma}{3}} C_{\infty}^{1+\gamma}}{3 I^{\frac{2-\gamma}{3}}}-\frac{a b \lambda^{\frac{1+2 \sigma}{3}} C_{\infty} S_{\infty}^{\sigma}}{3 I^{\frac{2-2 \sigma}{3}}} \\
& -\frac{a b \lambda^{\frac{2 \sigma+3+\gamma}{3}} C_{\infty}^{1+\gamma} S_{\infty}^{\sigma}}{3 I^{\frac{2-2 \sigma-\gamma}{3}}}-\frac{\lambda^{\frac{2}{3}} C_{\infty}^{2} \varepsilon}{3 I^{\frac{1}{3}}},
\end{aligned}
$$

where $p_{\infty}=\max _{t \in[0,2 \pi]}|p(t)|$. Thus, in view of $0<\sigma<\frac{1}{2}, 0<\gamma<1$, and $2 \sigma+\gamma<1$, Lemma 2.2, and (i), there exists $\bar{I}_{1}>0$ such that $\frac{d \theta}{d t}>0$ if $I_{0} \geq \bar{I}_{1}$.

In the case $|x| \leq M$, we may assume that $f_{\infty}=\max _{|x| \leq M}|f(x)|, \psi_{\infty}=\max _{|x| \leq M}|\psi(x)|$, then by Remark 1.3, we get

$$
\begin{aligned}
\frac{d \theta}{d t} & =\frac{1}{3} \lambda^{\frac{4}{3}} \alpha I^{\frac{1}{3}}+\frac{x(f(x) q(y)+\psi(x)-p(t))}{3 I} \\
& \geq \frac{1}{3} \lambda^{\frac{4}{3}} \alpha I^{\frac{1}{3}}-\frac{M f_{\infty} q(y)}{3 I}-\frac{\left(\psi_{\infty}+p_{\infty}\right) M}{3 I} \\
& \geq \frac{1}{3} \lambda^{\frac{4}{3}} \alpha I^{\frac{1}{3}}-\frac{M b f_{\infty} \lambda^{\frac{2 \sigma}{3}}}{3 I^{1-2 \sigma}}-\frac{\left(b f_{\infty}+\psi_{\infty}+p_{\infty}\right) M}{3 I} .
\end{aligned}
$$

So, by $0<\sigma<\frac{1}{2}$, (2.3), and Lemma 2.2, there exists a constant $\bar{I}_{2}>0$, such that $\frac{d \theta}{d t}>0$ if $I_{0} \geq \bar{I}_{2}$.

If we take $\bar{I}=\max \left\{\bar{I}_{1}, \bar{I}_{2}\right\}$, then $I_{0} \geq \bar{I}$ implies $\frac{d \theta}{d t}>0$.

\section{Twist property and proof of Theorem 1.1}

In this section, we will prove the existence of Aubry-Mather sets and quasi-periodic solutions of (1.1) via the Aubry-Mather theory for reversible systems developed by Chow and Pei [8].

In order to fit into the framework of Aubry-Mather theory for reversible systems, we only need to show that the Poincaré map $P$ has the monotone twist property around infinity, i.e. $\frac{\partial \theta\left(2 \pi ; \theta_{0}, I_{0}\right)}{\partial I_{0}}<0$ if $I_{0} \gg 1$.

In the following, we will investigate the behavior of $\frac{\partial \theta\left(2 \pi ; \theta_{0}, I_{0}\right)}{\partial I_{0}}$ when $I_{0} \gg 1$ by some lemmas.

Similarly, for the sake of convenience in later discussions we write $x, y, \theta, I$ instead of $x\left(\theta\left(t ; \theta_{0}, I_{0}\right), I\left(t ; \theta_{0}, I_{0}\right)\right), y\left(\theta\left(t ; \theta_{0}, I_{0}\right), I\left(t ; \theta_{0}, I_{0}\right)\right), \theta\left(t ; \theta_{0}, I_{0}\right), I\left(t ; \theta_{0}, I_{0}\right)$, respectively.

Lemma 3.1 The following limits exist uniformly on $t \in[0,2 \pi]$ : 
(i) $\frac{x \psi(x)}{I^{\frac{4}{3}}} \rightarrow 0 ; \frac{x^{3} \psi(x)}{I^{\frac{4}{3}}} \rightarrow 0 ; \frac{x^{2} \psi^{\prime}(x)}{I^{\frac{4}{3}}} \rightarrow 0$, as $I_{0} \rightarrow+\infty$;

(ii) $\frac{y \psi(x)}{I^{\frac{4}{3}}} \rightarrow 0 ; \frac{y x \psi^{\prime}(x)}{I^{\frac{4}{3}}} \rightarrow 0 ; \frac{y^{2} \psi^{\prime}(x)}{I^{\frac{4}{3}}} \rightarrow 0$, as $I_{0} \rightarrow+\infty$.

Proof From Remark 1.1, we note that $\lim _{|x| \rightarrow+\infty} \psi^{\prime}(x)=0$ and $\lim _{|x| \rightarrow+\infty} \frac{\psi(x)}{x}=0$. So, given any $\varepsilon>0$, there is a positive number $M=M(\varepsilon)>0$, such that $|x| \geq M$ imply

$$
\left|\psi^{\prime}(x)\right| \leq \varepsilon
$$

and

$$
|\psi(x)| \leq \varepsilon|x|
$$

for $\forall t \in[0,2 \pi]$.

Let $K_{1}(\varepsilon)=\max _{|x| \leq M}|\psi(x)|, K_{2}(\varepsilon)=\max _{|x| \leq M}\left|\psi^{\prime}(x)\right|$.

(i) According to (2.3) and Lemma 2.1(iv), one has

$$
\begin{aligned}
\left|\frac{x \psi(x)}{I^{\frac{4}{3}}}\right| & \leq \frac{M K_{1}(\varepsilon)}{I^{\frac{4}{3}}}+\frac{\varepsilon x^{2}}{I^{\frac{4}{3}}} \leq \frac{M K_{1}(\varepsilon)}{I^{\frac{4}{3}}}+\frac{\varepsilon(\lambda I)^{\frac{2}{3}} C_{\infty}^{2}}{I^{\frac{4}{3}}} \\
& \leq \frac{M K_{1}(\varepsilon)}{I^{\frac{4}{3}}}+\frac{\varepsilon \lambda^{\frac{2}{3}}}{I^{\frac{2}{3}}} ; \\
\left|\frac{x^{3} \psi(x)}{I^{\frac{4}{3}}}\right| & \leq \frac{M^{3} K_{1}(\varepsilon)}{I^{\frac{4}{3}}}+\frac{\varepsilon x^{4}}{I^{\frac{4}{3}}} \leq \frac{M^{3} K_{1}(\varepsilon)}{I^{\frac{4}{3}}}+\frac{\varepsilon(\lambda I)^{\frac{4}{3}} C_{\infty}^{4}}{I^{\frac{4}{3}}} \\
& \leq \frac{M^{3} K_{1}(\varepsilon)}{I^{\frac{4}{3}}}+\varepsilon C_{\infty}^{4} \lambda^{\frac{4}{3}} ; \\
\left|\frac{x^{2} \psi^{\prime}(x)}{I^{\frac{4}{3}}}\right| & \leq \frac{M^{2} K_{2}(\varepsilon)}{I^{\frac{4}{3}}}+\frac{\varepsilon x^{2}}{I^{\frac{4}{3}}} \leq \frac{M^{2} K_{2}(\varepsilon)}{I^{\frac{4}{3}}}+\frac{\varepsilon\left(\lambda I^{\frac{2}{3}} C_{\infty}^{2}\right.}{I^{\frac{4}{3}}} \\
& \leq \frac{M^{2} K_{2}(\varepsilon)}{I^{\frac{4}{3}}}+\frac{\varepsilon C_{\infty}^{2} \lambda^{\frac{2}{3}}}{I^{\frac{2}{3}}} .
\end{aligned}
$$

Then, by using Lemma 2.3(i), given $\bar{I}>0$, choose $I_{0}$ so that $I_{0} \geq \bar{I}$, provided

$$
I(t)^{\frac{4}{3}}>\max \left\{\frac{M K_{1}(\varepsilon)}{\varepsilon} ; \frac{M^{3} K_{1}(\varepsilon)}{\varepsilon} ; \frac{M^{2} K_{2}(\varepsilon)}{\varepsilon} ; 1\right\}
$$

we have

$$
\left|\frac{x \psi(x)}{I^{\frac{4}{3}}}\right|<\varepsilon+\lambda^{\frac{2}{3}} \varepsilon ; \quad\left|\frac{x^{3} \psi(x)}{I^{\frac{4}{3}}}\right|<\varepsilon+\lambda^{\frac{4}{3}} C_{\infty}^{4} \varepsilon ; \quad\left|\frac{x^{2} \psi^{\prime}(x)}{I^{\frac{4}{3}}}\right|<\varepsilon+\lambda^{\frac{2}{3}} C_{\infty}^{2} \varepsilon .
$$

Since $\varepsilon>0$ is arbitrary the proof of (i) is complete.

(ii) By (2.3), $\left(\mathrm{H}_{3}\right)$, Lemma 2.1(iv), and $\psi^{\prime}(0)=0$, it follows that

$$
\begin{aligned}
\left|\frac{y \psi(x)}{I^{\frac{4}{3}}}\right| & \leq \frac{|y| K_{1}(\varepsilon)}{I^{\frac{4}{3}}}+\frac{\varepsilon|y||x|}{I^{\frac{4}{3}}} \leq \frac{(\lambda I)^{\frac{2}{3}} S_{\infty} K_{1}(\varepsilon)}{I^{\frac{4}{3}}}+\frac{\varepsilon \lambda I C_{\infty} S_{\infty}}{I^{\frac{4}{3}}} \\
& \leq \frac{\lambda^{\frac{2}{3}} S_{\infty} K_{1}(\varepsilon)}{I^{\frac{2}{3}}}+\frac{\lambda C_{\infty} S_{\infty} \varepsilon}{I^{\frac{1}{3}}}
\end{aligned}
$$




$$
\begin{aligned}
\left|\frac{y x \psi^{\prime}(x)}{I^{\frac{4}{3}}}\right| & \leq \frac{\mu|y|}{I^{\frac{4}{3}}} \leq \frac{\mu(\lambda I)^{\frac{2}{3}} S_{\infty}}{I^{\frac{4}{3}}} \leq \frac{\mu \lambda^{\frac{2}{3}} S_{\infty}}{I^{\frac{2}{3}}} \\
\left|\frac{y^{2} \psi^{\prime}(x)}{I^{\frac{4}{3}}}\right| & \leq\left|\frac{y^{2} \psi^{\prime}(0)}{I^{\frac{4}{3}}}\right|+\frac{y^{2}\left|x \psi^{\prime}(x)\right|}{|x| I^{\frac{4}{3}}} \leq \frac{y^{2}\left|x \psi^{\prime}(x)\right|}{|x| I^{\frac{4}{3}}}
\end{aligned}
$$

so we have

$$
\left|\frac{y^{2} \psi^{\prime}(x)}{I^{\frac{4}{3}}}\right| \leq \frac{y^{2}\left|x \psi^{\prime}(x)\right|}{I^{\frac{4}{3}}(\lambda I)^{\frac{1}{3}} C_{\infty}} \leq \frac{(\lambda I)^{\frac{4}{3}} S_{\infty}^{2} \mu}{I^{\frac{4}{3}}(\lambda I)^{\frac{1}{3}} C_{\infty}}=\frac{\lambda S_{\infty}^{2} \mu}{I^{\frac{1}{3}} C_{\infty}} \leq \frac{\lambda S_{\infty}^{2} \mu}{I^{\frac{1}{3}} C_{\infty}} .
$$

Consequently, by using Lemma 2.3(i), given $\bar{I}>0$, choose $I_{0}$ so that $I_{0} \geq \bar{I}$, provided

$$
I(t)^{\frac{2}{3}}>\max \left\{\frac{\lambda^{\frac{2}{3}} S_{\infty} K_{1}(\varepsilon)}{\varepsilon} ; \frac{\mu \lambda^{\frac{2}{3}} S_{\infty}}{\varepsilon} ;\left(\frac{\lambda S_{\infty}^{2} \mu}{C_{\infty} \varepsilon}\right)^{2} ; 1\right\}
$$

we have

$$
\left|\frac{y \psi(x)}{I^{\frac{4}{3}}}\right|<\left(1+\lambda C_{\infty} S_{\infty}\right) \varepsilon ; \quad\left|\frac{y x \psi^{\prime}(x)}{I^{\frac{4}{3}}}\right|<\varepsilon ; \quad\left|\frac{y^{2} \psi^{\prime}(x)}{I^{\frac{4}{3}}}\right|<\varepsilon
$$

Since $\varepsilon>0$ is arbitrary, (ii) is proved.

Lemma 3.2 The following limits hold uniformly over $t \in[0,2 \pi]$ :

(i) $\frac{y q^{\prime}(y) x f(x)}{I^{\frac{4}{3}}} \rightarrow 0 ; \frac{y q(y) f(x)}{I^{\frac{4}{3}}} \rightarrow 0 ; \frac{q(y) f(x) x^{3}}{I^{\frac{4}{3}}} \rightarrow 0$, as $I_{0} \rightarrow+\infty$;

(ii) $\frac{y q(y) x f^{\prime}(x)}{I^{\frac{4}{3}}} \rightarrow 0 ; \frac{q(y) x^{2} f^{\prime}(x)}{I^{\frac{4}{3}}} \rightarrow 0 ; \frac{y^{2} q(y) f^{\prime}(x)}{I^{\frac{4}{3}}} \rightarrow 0 ; \frac{q^{\prime}(y) f(x) x^{4}}{I^{\frac{4}{3}}} \rightarrow 0$, as $I_{0} \rightarrow+\infty$.

Proof (i) By Remark 1.2, Remark 1.3, (2.3), and Lemma 2.1(iv), we know that

$$
\begin{aligned}
& \left|\frac{y q^{\prime}(y) x f(x)}{I^{\frac{4}{3}}}\right| \leq \frac{a b\left(1+|y|^{\sigma}\right)\left(1+|x|^{\gamma}\right)}{I^{\frac{4}{3}}} \\
& \leq \frac{a b}{I^{\frac{4}{3}}}+\frac{a b \lambda^{\frac{\gamma}{3}} C_{\infty}^{\gamma}}{I^{\frac{4-\gamma}{3}}}+\frac{a b \lambda^{\frac{2 \sigma}{3}} S_{\infty}^{\sigma}}{I^{\frac{4-2 \sigma}{3}}}+\frac{a b \lambda^{\frac{2 \sigma+\gamma}{3}} C_{\infty}^{\gamma} S_{\infty}^{\sigma}}{I^{\frac{4-2 \sigma-\gamma}{3}}} ; \\
& \left|\frac{y q(y) f(x)}{I^{\frac{4}{3}}}\right| \leq \frac{a b|y|\left(1+|y|^{\sigma}\right)\left(1+|x|^{\gamma}\right)}{I^{\frac{4}{3}}} \\
& \leq \frac{a b \lambda^{\frac{2}{3}} S_{\infty}}{I^{\frac{2}{3}}}+\frac{a b \lambda^{\frac{2+\gamma}{3}} S_{\infty} C_{\infty}^{\gamma}}{I^{\frac{2-\gamma}{3}}}+\frac{a b \lambda^{\frac{2+2 \sigma}{3}} S_{\infty}^{\sigma+1}}{I^{\frac{2-2 \sigma}{3}}}+\frac{a b \lambda^{\frac{2+2 \sigma+\gamma}{3}} C_{\infty}^{\gamma} S_{\infty}^{\sigma+1}}{I^{\frac{2-2 \sigma-\gamma}{3}}} \\
& \left|\frac{q(y) f(x) x^{3}}{I^{\frac{4}{3}}}\right| \leq \frac{a b\left(1+|y|^{\sigma}\right)\left(1+|x|^{\gamma}\right)|x|^{3}}{I^{\frac{4}{3}}} \\
& \leq \frac{a b \lambda C_{\infty}^{3}}{I^{\frac{1}{3}}}+\frac{a b \lambda^{\frac{\sigma+3}{3}} S_{\infty}^{\sigma} C_{\infty}^{3}}{I^{\frac{1-\gamma}{3}}}+\frac{a b \lambda^{\frac{3+\gamma}{3}} C_{\infty}^{3+\gamma}}{I^{\frac{1-2 \sigma}{3}}}+\frac{a b \lambda^{\frac{2 \sigma+\gamma+3}{3}} C_{\infty}^{\gamma+3} S_{\infty}^{\sigma}}{I^{\frac{1-2 \sigma-\gamma}{3}}} .
\end{aligned}
$$

Noting that $0<\gamma<1,0<\sigma<\frac{1}{2}$, and $2 \sigma+\gamma<1$, it follows that the conclusions of (i) are established by virtue of Lemma 2.2 and Lemma 2.3(i) as $I_{0} \gg 1$. 
(ii) From $f^{\prime}(0)=q^{\prime}(0)=0$, Remark 1.2, Remark 1.3, (2.3), and Lemma 2.1(iv), we get

$$
\begin{aligned}
\left|\frac{y q(y) x f^{\prime}(x)}{I^{\frac{4}{3}}}\right| & \leq \frac{a b|y|\left(1+|y|^{\sigma}\right)\left(1+|x|^{\gamma}\right)}{I^{\frac{4}{3}}} \\
& \leq \frac{a b \lambda^{\frac{2}{3}} S_{\infty}}{I^{\frac{2}{3}}}+\frac{a b \lambda^{\frac{2+\gamma}{3}} S_{\infty} C_{\infty}^{\gamma}}{I^{\frac{2-\gamma}{3}}}+\frac{a b \lambda^{\frac{2+2 \sigma}{3}} S_{\infty}^{\sigma+1}}{I^{\frac{2-2 \sigma}{3}}}+\frac{a b \lambda^{\frac{2+2 \sigma+\gamma}{3}} C_{\infty}^{\gamma} S_{\infty}^{\sigma+1}}{I^{\frac{2-2 \sigma-\gamma}{3}}} \\
\left|\frac{q(y) x^{2} f^{\prime}(x)}{I^{\frac{4}{3}}}\right| & \leq \frac{a b\left(1+|y|^{\sigma}\right)\left(1+|x|^{\gamma}\right)|x|}{I^{\frac{4}{3}}} \\
& \leq \frac{a b \lambda^{\frac{1}{3}} C_{\infty}}{I^{2}}+\frac{a b \lambda^{\frac{3+\gamma}{3}} C_{\infty}^{1+\gamma}}{I^{\frac{3-\gamma}{3}}}+\frac{a b \lambda^{\frac{1+2 \sigma}{3}} C_{\infty} S_{\infty}^{\sigma}}{I^{\frac{3-2 \sigma}{3}}}+\frac{a b \lambda^{\frac{2 \sigma+3+\gamma}{3}} C_{\infty}^{1+\gamma} S_{\infty}^{\sigma}}{I^{\frac{3-2 \sigma-\gamma}{3}}} \\
& \leq \frac{a b\left(1+|y|^{\sigma}\right)|y|^{2}\left(1+|x|^{\gamma}\right)}{|x| I^{\frac{4}{3}}} \\
\left|\frac{y^{2} q(y) f^{\prime}(x)}{I^{\frac{4}{3}}}\right| & \leq \frac{\left|y^{2} q(y) \cdot f^{\prime}(0)\right|}{I^{\frac{4}{3}}}+\frac{\left|y^{2} q(y) x f^{\prime}(x)\right|}{|x| I^{\frac{4}{3}}} \\
& \leq \frac{a b\left(1+|y|^{\sigma}\right)\left(1+|x|^{\gamma}\right)|x|^{4}}{|y| I^{\frac{4}{3}}}, \\
\left|\frac{q^{\prime}(y) f(x) x^{4}}{I^{\frac{4}{3}}}\right| & \leq\left|\frac{q^{\prime}(0) f(x) x^{4}}{I^{\frac{4}{3}}}\right|+\frac{\left|y q^{\prime}(y) f(x) x^{4}\right|}{|y| I^{\frac{4}{3}}}
\end{aligned}
$$

then we further obtain

$$
\begin{aligned}
\left|\frac{y^{2} q(y) f^{\prime}(x)}{I^{\frac{4}{3}}}\right| \leq & \frac{a b\left(1+|y|^{\sigma}\right)|y|^{2}\left(1+|x|^{\gamma}\right)}{\lambda^{\frac{1}{3}} I^{\frac{5}{3}} C_{\infty}} \\
\leq & \frac{a b \lambda C_{\infty}^{-1} S_{\infty}^{2}}{I^{\frac{1}{3}}}+\frac{a b \lambda^{\frac{3+\gamma}{3}} C_{\infty}^{\gamma-1} S_{\infty}^{2}}{I^{\frac{1-\gamma}{3}}}+\frac{a b \lambda^{\frac{3+2 \sigma}{3}} C_{\infty}^{-1} S_{\infty}^{2+\sigma}}{I^{\frac{1-2 \sigma}{3}}} \\
& +\frac{a b \lambda^{\frac{3+2 \sigma+\gamma}{3}} C_{\infty}^{\gamma-1} S_{\infty}^{2+\sigma}}{I^{\frac{1-2 \sigma-\gamma}{3}}} ; \\
\left|\frac{q^{\prime}(y) f(x) x^{4}}{I^{\frac{4}{3}} \mid \leq}\right| & \frac{a b\left(1+|y|^{\sigma}\right)\left(1+|x|^{\gamma}\right)|x|^{4}}{I^{\frac{4}{3}} \lambda^{\frac{2}{3}} I^{\frac{2}{3}} S_{\infty}} \\
\leq & \frac{a b \lambda^{\frac{2}{3}} C_{\infty}^{4} S_{\infty}^{-1}}{I^{\frac{2}{3}}+\frac{a b \lambda^{\frac{2+\gamma}{3}} C_{\infty}^{4+\gamma} S_{\infty}^{-1}}{I^{\frac{2-\gamma}{3}}}+\frac{a b \lambda^{\frac{2+2 \sigma}{3}} C_{\infty}^{4} S_{\infty}^{\sigma-1}}{I^{\frac{2-2 \sigma}{3}}}} \\
& +\frac{a b \lambda^{\frac{2 \sigma+2+\gamma}{3}} C_{\infty}^{4+\gamma} S_{\infty}^{\sigma-1}}{I^{\frac{2-2 \sigma-\gamma}{3}}} .
\end{aligned}
$$

In the same way, by the facts $0<\gamma<1,0<\sigma<\frac{1}{2}$, and $2 \sigma+\gamma<1$, we can draw the conclusions of (ii) in view of Lemma 2.2 and Lemma 2.3(i) as $I_{0} \gg 1$.

For any $t \in[0,2 \pi]$, we put

$$
\begin{aligned}
a_{1}(t) & =\frac{\partial \Phi_{1}}{\partial I} \\
& =\frac{1}{9} \alpha \lambda^{\frac{4}{3}} I^{-\frac{2}{3}}-\frac{x\left(2(\psi(x)-p(t))-x \psi^{\prime}(x)\right)}{9 I^{2}}+\frac{x^{2} f^{\prime}(x) q(y)}{9 I^{2}}+\frac{2 x f(x)\left(y q^{\prime}(y)-q(y)\right)}{9 I^{2}}
\end{aligned}
$$




$$
\begin{aligned}
a_{2}(t) & =\frac{\partial \Phi_{1}}{\partial \theta} \\
& =\frac{y\left(x \psi^{\prime}(x)+\psi(x)-p(t)\right)}{3 \omega \lambda^{\frac{1}{3}} I^{\frac{4}{3}}}+\frac{y q(y)\left(f(x)+x f^{\prime}(x)\right)}{3 \omega \lambda^{\frac{1}{3}} I^{\frac{4}{3}}}+\frac{q^{\prime}(y) f(x)\left(\alpha x^{+4}+\beta x^{-4}\right)}{3 \omega \lambda^{\frac{1}{3}} I^{\frac{4}{3}}} \\
a_{3}(t) & =\frac{\partial \Phi_{2}}{\partial I} \\
& =-\frac{y\left(x \psi^{\prime}(x)+\psi(x)-p(t)\right)}{3 \omega \lambda^{\frac{1}{3}} I^{\frac{4}{3}}}-\frac{y q(y)\left(f(x)+x f^{\prime}(x)\right)+2 y^{2} q^{\prime}(y) f(x)}{3 \omega \lambda^{\frac{1}{3}} I^{\frac{4}{3}}} \\
a_{4}(t) & =\frac{\partial \Phi_{2}}{\partial \theta} \\
& =\frac{\left(\beta x^{-3}-\alpha x^{+3}\right)\left(\psi(x)-p(t)+f(x) q(y)+y f(x) q^{\prime}(y)\right)}{\omega^{2}(\lambda I)^{\frac{2}{3}}}+\frac{y^{2}\left(\psi^{\prime}(x)+f^{\prime}(x) q(y)\right)}{\omega^{2}(\lambda I)^{\frac{2}{3}}} .
\end{aligned}
$$

As a consequence of Lemma 2.3(i), Lemma 3.1, and Lemma 3.2, we have the following.

Lemma 3.3 For $I_{0}$ large enough and $\forall t, s \in[0,2 \pi]$, the following conclusions hold:

(i) $a_{1}(t)=o\left(\frac{1}{I_{0}^{\frac{1}{3}}}\right)$;

(ii) $a_{2}(t)=o(1), a_{3}(t)=o(1)$;

(iii) $a_{1}(t) \cdot a_{4}(s)=o(1)$.

For $\forall t \in[0,2 \pi]$, consider the variational equation of (2.4) with respect to the initial value $I_{0}$, we have

$$
\dot{\theta}_{I_{0}}=a_{1}(t) \frac{\partial I}{\partial I_{0}}+a_{2}(t) \frac{\partial \theta}{\partial I_{0}}, \quad \dot{I}_{I_{0}}=a_{3}(t) \frac{\partial I}{\partial I_{0}}+a_{4}(t) \frac{\partial \theta}{\partial I_{0}} .
$$

Combining the previous estimates, we have the following.

Lemma 3.4 For all $t \in(0,2 \pi], I_{0} \rightarrow+\infty$, the following conclusions hold true:

(i) $\theta_{I_{0}}\left(t ; \theta_{0}, I_{0}\right) \rightarrow 0$;

(ii) $I_{I_{0}}\left(t ; \theta_{0}, I_{0}\right)=1+o(1)$;

(iii) $\theta_{\theta_{0}}\left(t ; \theta_{0}, I_{0}\right)=1+o(1)$.

Proof From the variational equations (3.1) and Lemma 3.3, one has

$$
\begin{aligned}
\theta_{I_{0}}(t) & =e^{\int_{0}^{t} a_{2}(s) d s} \int_{0}^{t} e^{-\int_{0}^{s} a_{2}(t) d t} a_{1}(s) I_{I_{0}}(s) d s \\
& =(1+o(1)) \int_{0}^{t} a_{1}(s) I_{I_{0}}(s) d s ; \\
I_{I_{0}}(t) & =e^{\int_{0}^{t} a_{3}(s) d s}\left(1+\int_{0}^{t} e^{-\int_{0}^{s} a_{3}(t) d t} a_{4}(s) \theta_{I_{0}}(s) d s\right) \\
& =1+o(1)+(1+o(1)) \int_{0}^{t} a_{4}(s)\left(\int_{0}^{s} a_{1}(t) I_{I_{0}}(t) d t\right) d s \\
& =1+o(1)+o(1) \int_{0}^{t} \int_{0}^{s} I_{I_{0}}(t) d t d s .
\end{aligned}
$$

Hence, for all $t \in(0,2 \pi]$, as $I_{0} \rightarrow+\infty$, we have $I_{I_{0}}(t)=1+o(1)$ and $\theta_{I_{0}}(t)=(1+$ $o(1)) \int_{0}^{t} a_{1}(s) d s \rightarrow 0$. Thus, (i) and (ii) are proved. 
To verify (iii), considering the variational equation of (2.4) about $\theta_{0}$, one obtains

$$
\dot{\theta}_{\theta_{0}}=a_{1}(t) \frac{\partial I}{\partial \theta_{0}}+a_{2}(t) \frac{\partial \theta}{\partial \theta_{0}}, \quad \dot{I}_{\theta_{0}}=a_{3}(t) \frac{\partial I}{\partial \theta_{0}}+a_{4}(t) \frac{\partial \theta}{\partial \theta_{0}} .
$$

Using similar arguments in (ii), we deduce that $\theta_{\theta_{0}}\left(t ; \theta_{0}, I_{0}\right)=1+o(1)$ for $\forall t \in(0,2 \pi]$, as $I_{0} \rightarrow+\infty$. This completes the proof of Lemma 3.4.

The following lemma gives an estimate of lower bound for $a_{1}(t)$.

Lemma 3.5 For all $t \in[0,2 \pi]$ and $I_{0}$ large enough, there exists a constant $L_{d}>0$, such that

$$
\left|a_{1}(t)\right| \geq \frac{L_{d}}{I^{\frac{2}{3}}(t)}
$$

Moreover,

$$
a_{1}(t)>0 \text {. }
$$

Proof According to condition $\left(\mathrm{H}_{3}\right)$, we can assume

$$
|\psi(x)| \leq \mu|x|+c_{1}
$$

where $c_{1}>0$ is a constant. Then, by $\left(\mathrm{H}_{3}\right)$ and $(2.3)$, we have

$$
\left|\frac{x\left(2(\psi(x)-p(t))-x \psi^{\prime}(x)\right)}{9 I^{2}}\right| \leq\left|\frac{2 \mu \lambda^{\frac{2}{3}} C_{\infty}^{2}}{9 I^{\frac{4}{3}}}+\frac{\left(2 c_{1}+p_{\infty}+\mu\right) \lambda^{\frac{1}{3}} C_{\infty}}{9 I^{\frac{5}{3}}}\right|:=a_{11}(I) .
$$

Similar to the proof of Lemma 3.2, we have

$$
\begin{gathered}
\left|\frac{x^{2} f^{\prime}(x) q(y)}{9 I^{2}}\right| \leq\left|\frac{a b \lambda^{\frac{1}{3}} C_{\infty}}{9 I^{\frac{5}{3}}}+\frac{a b \lambda^{\frac{3+\gamma}{3}} C_{\infty}^{1+\gamma}}{9 I^{\frac{5-\gamma}{3}}}+\frac{a b \lambda^{\frac{1+2 \sigma}{3}} C_{\infty} S_{\infty}^{\sigma}}{9 I^{\frac{5-2 \sigma}{3}}}+\frac{a b \lambda^{\frac{2 \sigma+3+\gamma}{3}} C_{\infty}^{1+\gamma} S_{\infty}^{\sigma}}{9 I^{\frac{5-2 \sigma-\gamma}{3}}}\right| \\
:=a_{12}(I) ; \\
\left|\frac{2 x f(x)\left(y q^{\prime}(y)-q(y)\right)}{9 I^{2}}\right| \leq\left|\frac{4 a b}{9 I^{2}}+\frac{4 a b \lambda^{\frac{\gamma}{3}} C_{\infty}^{\gamma}}{9 I^{\frac{6-\gamma}{3}}}+\frac{4 a b \lambda^{\frac{2 \sigma}{3}} S_{\infty}^{\sigma}}{9 I^{\frac{6-2 \sigma}{3}}}+\frac{4 a b \lambda^{\frac{2 \sigma+\gamma}{3}} C_{\infty}^{\gamma} S_{\infty}^{\sigma}}{9 I^{\frac{6-2 \sigma-\gamma}{3}}}\right| \\
:=a_{13}(I) .
\end{gathered}
$$

By $0<\gamma<1,0<\sigma<\frac{1}{2}, 2 \sigma+\gamma<1$, and Lemma 2.3(i), we can choose $I_{0}$ large enough so that

$$
\frac{\alpha \lambda^{\frac{4}{3}}}{18 I^{\frac{2}{3}}}-a_{11}(I)-a_{12}(I)-a_{13}(I) \geq 0
$$

Hence

$$
a_{1}(t)>0 \text {. }
$$


Further,

$$
\begin{aligned}
\left|a_{1}(t)\right|= & \mid \frac{1}{9} \alpha \lambda^{\frac{4}{3}} I^{-\frac{2}{3}}-\frac{x\left(2(\psi(x)-p(t))-x \psi^{\prime}(x)\right)}{9 I^{2}} \\
& +\frac{x^{2} f^{\prime}(x) q(y)}{9 I^{2}}+\frac{2 x f(x)\left(y q^{\prime}(y)-q(y)\right)}{9 I^{2}} \mid \\
\geq & \frac{\alpha \lambda^{\frac{4}{3}}}{18 I^{\frac{2}{3}}}+\frac{\alpha \lambda^{\frac{4}{3}}}{18 I^{\frac{2}{3}}}-a_{11}(I)-a_{12}(I)-a_{13}(I) \\
\geq & \frac{\lambda^{\frac{4}{3}} \alpha}{18 I^{\frac{2}{3}}(t)} .
\end{aligned}
$$

Thus, if we take $L_{d}=\frac{\lambda^{\frac{4}{3}} \alpha}{18}$, we see $\left|a_{1}(t)\right| \geq \frac{L_{d}}{I^{\frac{2}{3}}(t)}$.

Therefore, combining the above discussions and Lemma 2.3(i), we see that

$$
\theta_{I_{0}}(2 \pi)=(1+o(1)) \int_{0}^{2 \pi} a_{1}(s) d s \geq(1+o(1)) \frac{2 \pi L_{d}}{\left(k_{2} I_{0}\right)^{\frac{2}{3}}}>0
$$

\section{if $I_{0}$ large enough.}

Hence, the Poincaré map has the monotone twist property for all $I_{0}$ large enough. This completes the proof of Theorem 1.1.

\section{Competing interests}

The author declares that they have no competing interests.

\section{Acknowledgements}

This work is supported by the Natural Science Foundation of China (No. 11461056), Natural Science Foundation of Jiangxi Province (20132BAB211008).

Received: 5 January 2015 Accepted: 5 June 2015 Published online: 18 June 2015

\section{References}

1. Pei, ML: Mather sets for superlinear Duffing's equations. Sci. China Math. 36(5), 524-537 (1993)

2. Qian, DB: Mather sets for sublinear Duffing equations. Chin. Ann. Math., Ser. B 15(1), 421-434 (1994)

3. Pei, ML: Aubry-Mather sets for finite-twist maps of a cylinder and semilinear Duffing equations. J. Differ. Equ. 113(1), 106-127 (1994)

4. Capietto, A, Liu, B: Quasi-periodic solutions of a forced asymmetric oscillator at resonance. Nonlinear Anal. 56, 105-117 (2004)

5. Liu, B: Boundedness in nonlinear oscillations at resonance. J. Differ. Equ. 153, 142-154 (1999)

6. Capietto, A, Dambrosio, W, Liu, B: On the boundedness of solutions to a nonlinear singular oscillator. Z. Angew. Math. Phys. 60(6), 1007-1034 (2009)

7. Capietto, A, Dambrosio, W, Wang, XP: Quasi-periodic solutions of a damped reversible oscillator at resonance. Differ. Integral Equ. 22(9/10), 1033-1046 (2009)

8. Chow, SN, Pei, ML: Aubry-Mather theorem and quasiperiodic orbits for time dependent reversible systems. Nonlinear Anal., Theory Methods Appl. 25(9), 905-931 (1995)

9. Kunze, M, Kupper, T, Liu, B: Boundedness and unboundedness of solutions for reversible oscillators at resonance. Nonlinearity 14, 1105-1122 (2001)

10. Liu, B, Wang, R: Quasiperiodic solutions for reversible oscillators at resonance. Preprint

11. Wang, ZH, Wang, YQ, Li, H: Boundedness of solutions for Duffing equations with asymmetric superlinear restoring terms. Chin. Ann. Math., Ser. A 23(2), 187-196 (2002)

12. Yang, XJ: Boundedness of solutions for sublinear reversible systems. Appl. Math. Comput. 158, $389-396$ (2004)

13. Liu, B, Song, JJ: Invariant curved of reversible mappings with small twist. Acta Math. Sin. Engl. Ser. 20, 15-24 (2004)

14. Li, X: Invariant tori for semilinear reversible systems. Nonlinear Anal. 56, 133-146 (2004)

15. Liu, B: Quasi-periodic solutions of a semilinear Liénard equation at resonance. Sci. China Math. 48, 1234-1244 (2005)

16. Wang, XM: Aubry-Mather sets for a class of superlinear asymmetric Duffing equations. Chin. Ann. Math., Ser. A 33(3), 351-358 (2012)

17. Zhang, TT, Si, JG: Boundedness of solutions for a class of sublinear reversible oscillators with periodic forcing. Abstr. Appl. Anal. 2013, Article ID 246343 (2013)

18. Wang, XM: Aubry-Mather sets for semilinear Duffing equations. Acta Math. Sin. 52(3), 605-610 (2009)

19. Wang, XM: Aubry-Mather sets for sublinear asymmetric Duffing equations. Sci. China Math. 42(1), 13-21 (2012) 